\title{
Research and Application of Wireless Sensor Networks in Agriculture
}

\author{
Wei CAO ${ }^{\mathrm{a}}$, Jian $\mathrm{XU}^{\mathrm{b}, *}$, Juanjuan SHAN ${ }^{\mathrm{c}}$ Ruonan $\mathrm{SUN}^{\mathrm{d}}$ \\ Beijing University of Agriculture \\ BeiJing, China \\ E-mail: ${ }^{a} 979008969 @ q q . c o m$ \\ *E-mail: bjnxy@126.com \\ E-mail: ${ }^{c}$ loveyfb081115@qq.com \\ E-mail: ${ }^{\mathrm{d}} 597369409 @ q q . c o m$ \\ +* Corresponding author
}

\begin{abstract}
With the development of agricultural information technology, sensing technology, Internet communication technology and intelligent technology and agriculture. As an important technology in the Internet of things, wireless sensor networks (WSN) is an important technical form of the Internet of things. To explore the application of wireless sensor networks in agriculture and develop the application system of wireless sensor networks in agriculture has become a research hotspot. According to the actual characteristics of agriculture, and expounds the application in agriculture in the architecture of wireless sensor network, points out the existing problems, analyzes the development direction of agriculture in wireless sensor network, develop ideas for further application of wireless sensor networks in agriculture.
\end{abstract}

Keywords-wireless sensor networks; architecture; application progress

\section{INTRODUCTION}

As a new technology, wireless sensor network (WSN) originated in the United States military in 1970s, and the researchers have done a lot of research in the field of environmental monitoring, medical applications, industrial production monitoring and other fields. At present, there are few researches on the application of wireless sensor networks in the theory and application of facility agriculture at home and abroad.

In order to achieve high level of facility agricultural production and optimize the control of feeding biological environment, information acquisition is the key. In the field of agriculture, crop growth is affected by many natural conditions, such as temperature, humidity and light. The collection of information is very large, and the network can be used to realize the remote transmission of large amounts of data. However, if the laying of the cable network in the field of agriculture, on the one hand is not easy to cultivate, on the other hand, the cost is higher. In recent years, with the development of wireless sensor network, it provides a new idea and method for the monitoring of facility agriculture.

\section{A. Wireless Sensor Networks}

Wireless sensor networks will be the logical information world and the physical world together, will change the interaction between people and nature, with limited resources, dynamic network, data centric and high density of large number of nodes.

\section{B. Wireless Sensor Network Architecture}

The typical structure of wireless sensor network is shown in figure 1. Wireless sensor network system is composed of sensor nodes, sink nodes and task management nodes. Among them, a large number of sensor nodes are randomly or regularly arranged in the detection area, and the sensor nodes form a sensor network. The data collected by sensor nodes is processed by multi hop relay transmission to the sink node, and then through the external network (Internet, satellite, public mobile communication network, WLAN) is transmitted to the task management node, task management is the management of base station node, is actually a server and computer terminal. The manager can manage the base station and configure the sensor network to store, display and make decision on the collected data information, and can also publish the monitoring task or collect the data of the sensor network.

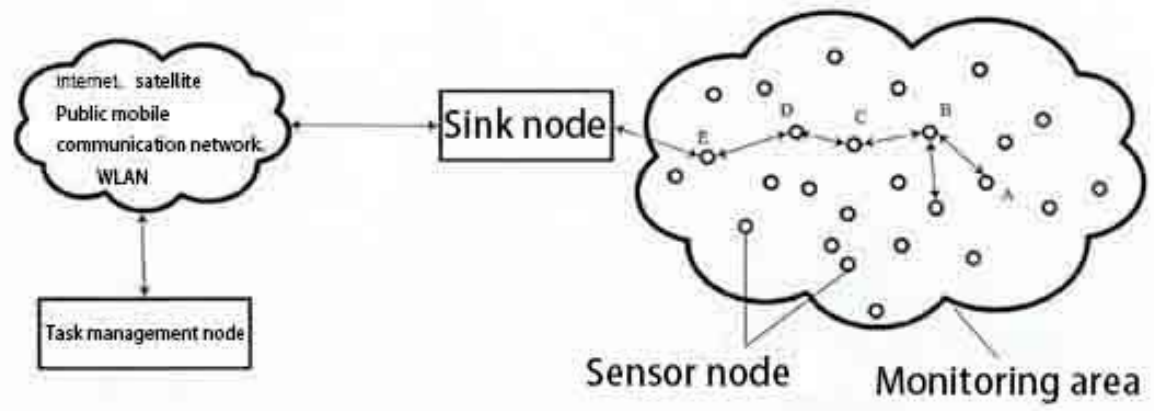

Figure 1. Typical structure of Wireless Sensor Networks 


\section{The formation process of Wireless Sensor Networks}

There are many ways to form wireless sensor networks, which can be divided into the following four steps. The representation of the formation process of the wireless sensor network is shown in figure 2 .

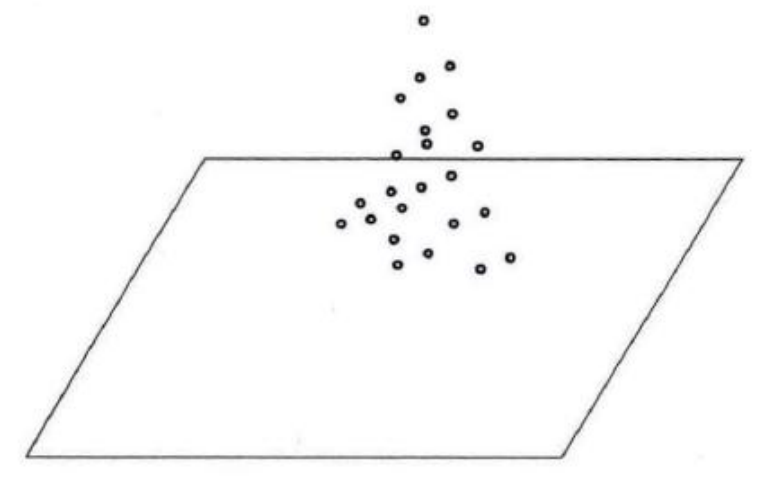

Step 1: deploy sensor nodes

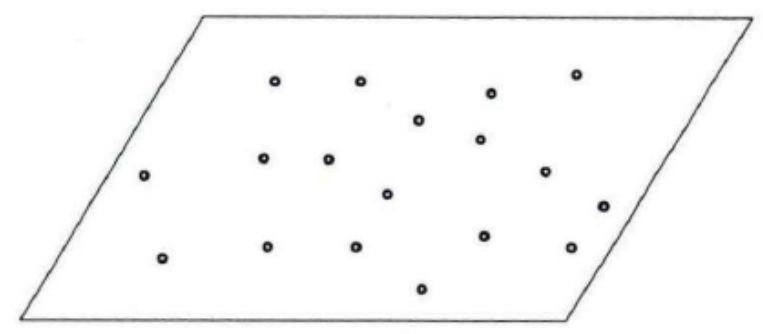

The second step: wake up and test each other

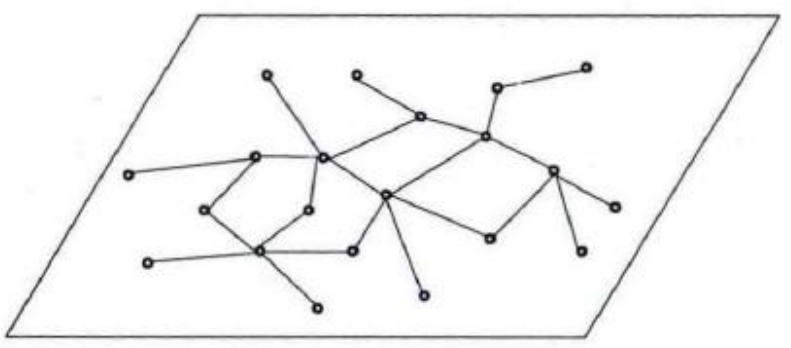

The third step: the formation of network

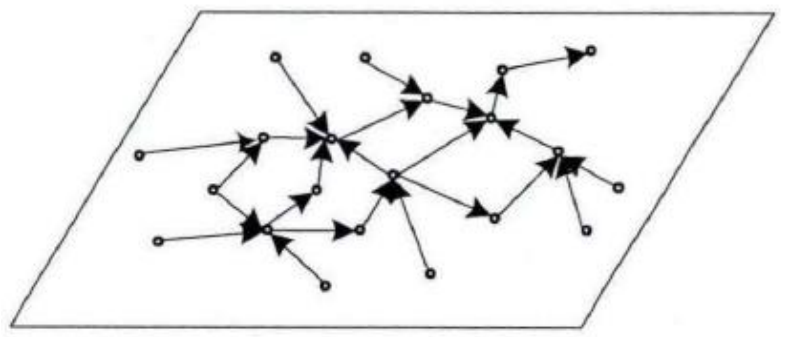

The fourth step: select the route for communication

Figure 2. Wireless sensor network formation process

Step 1: deploy sensor nodes. There are two ways to deploy the random and determine the way.

The second step: wake up and test each other. After the deployment of the sensor node is completed, the self start, and then enter the wake up state, all the sensor nodes can send signals to monitor and record the nearby sensor nodes.
The third step: the formation of network. After monitoring each other, the sensor nodes form a wireless network according to the monitoring results.

The fourth step: choose the route for communication. After the sensor nodes form a network, the routing algorithm is chosen for data communication.

\section{Network Protocol Architecture}

At present, the research focus of wireless sensor network is focused on the network architecture, including the MAC protocol, the network layer routing protocol and the node research. Research on network security robustness in network management module, network and node function consumption. The research of time synchronization, localization, multi-sensor information fusion and so on.

\section{E. Main Features Of Wireless Sensor Networks}

(1) Large network size and high node density. In order to obtain the accurate information, the sensor nodes will be deployed in the target area. The characteristics of wireless sensor networks have the following advantages: the large scale network to collect a large amount of information and distributed processing, which can improve the detection accuracy, without the requirement of each node has a very strong function. A large number of redundant nodes work together to improve the fault tolerance of the system, increase the coverage area and reduce the blind area. The signal to noise ratio is greater by acquiring information from different perspectives.

(2) No center, self-organization. Wireless sensor network is a peer-to-peer network, the status of each node is the same, there is no control center in the network. The nodes adjust their work by using the hierarchical protocol and the distributed algorithm, and then form the network rapidly.

(3) The energy of node power is limited, and the functions of communication, computation and storage are not very powerful. Sensor nodes are small, most of them use a very limited capacity of the battery, the exhaustion of energy indicates that the node loses its ability to work. Low power consumption is the primary consideration in hardware design.

(4) Strong dynamic. When the environment changes, or when the sensor node runs out of power failure and failure, environment change will cause changes in broadband wireless communication link, and may be disconnected and connected, in some cases also need to add new sensor nodes, so the network system with dynamic reconfiguration, when the topology changes, the structure can quickly change the sensor network, in order to respond to these changes.

(5) With data as the center, the direction of data transmission is strong. The traditional network is centered on the address, and the data is closely connected with the physical address of the node that drives the data. The wireless sensor network is based on the task of the network, people do not have to care about which node is monitored by the data, only need to pay attention to the monitoring area of the value of the monitoring indicators, that is, the data itself. 


\section{APPLICATION OF WIRELESS SENSOR NETWORKS IN AGRICULTURE}

At present, wireless technology is widely used in agriculture, but most of them are the application of the star topology structure of the base station. The general application of agriculture is a large number of sensor nodes monitoring network, information collected by various sensors, to help farmers to find problems, and to accurately determine the location of the problem that agriculture will be gradually taking human as the center, depending on the isolated mechanical production mode to the information and software centric mode of production thus, the extensive use of a variety of automated and intelligent remote control equipment.

\section{A. Greenhouse Environmental Applications}

In the greenhouse environment in a single greenhouse can become a wireless sensor network measurement and control area, nodes with different sensor nodes and has the advantages of simple actuator (actuator fan, motor, valves and other low voltage low current) composed of the wireless network to measure soil moisture, soil composition, $\mathrm{pH}$, temperature, and precipitation the air humidity and air pressure, light intensity and $\mathrm{CO}_{2}$ concentration to obtain the best conditions for crop growth, and the biological information acquisition method is applied to the wireless sensor nodes, and provide scientific basis for the precise regulation of greenhouse, the greenhouse in the sensors and actuators of standardized data, using the off network control device. In order to reach the scene convenient network, increase crop yield, improve quality, growth cycle, improve economic benefit adjustment.

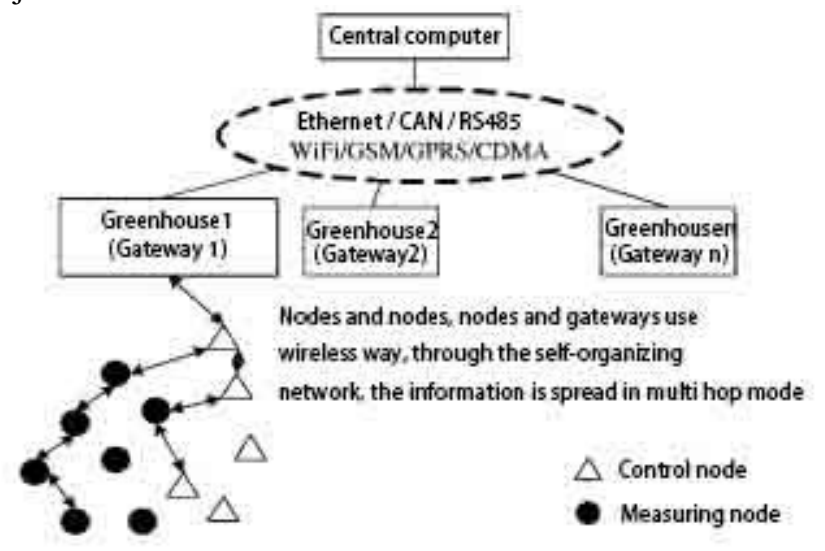

Figure 3. Wireless sensor network greenhouse application diagram

\section{B. Water Saving Irrigation Application}

Wireless sensor network node has the advantages of simple control, battery powered by power handling DC electromagnetic valve can control the different small and medium power (electric hydraulic solenoid valve, relief valve, pressure regulating valve, safety valve and flow control valve), dormancy state and the nodes of the network to work time if more than one year, powered by solar panels, energy does not need to think too much.

\section{Environmental Monitoring and Ecological Monitoring of Plants And Animals}

Through the wireless sensor network monitoring system layout of multiple levels, research on animal husbandry and poultry, aquaculture, life habit, rare animal environment and physiological conditions, can also be used for forest monitoring and fire alarm (usually nodes are random clouds in the forest, even under normal conditions regularly report the environmental data, when fire happens the node, through cooperation with the specific address, cooperation will fire in a very short period of time the size information transferred to the relevant departments).

\section{PROBLEMS AND DEVELOPMENT DIRECTION}

At present, various types of wireless sensor network monitoring system for facility agriculture environment based on the sensor are limited, mainly concentrated in the air temperature, air humidity, temperature and soil moisture, soil conductivity, carbon dioxide concentration, the water temperature water conductivity, through these sensor nodes, environmental information perception the network can only perceive the biological growth, not all of the environmental perception of biological information needed for growth, to further improve the accuracy of monitoring system. In addition, the existing agricultural facilities environment monitoring system based on wireless sensor network integration, energy management and acquired in the data management software, and also there is a serious shortage, directly influences the performance of the cognitive network.

In the future, with the continuous development of embedded technology, microelectronic technology and sensor technology, a new type of sensor node is developed, which enriches the types of sensor nodes and realizes the automatic control of more types of environmental information. The development of sensor nodes, the biological and physiological indexes (such as crop cell concentration, crop leaf water content, crop leaf temperature etc.) the real-time, remote and automatic control, and direct diagnosis of crop water stress and deficiency. At the same time, the establishment of safe and efficient wireless sensor network data fusion mechanism, the establishment of a safe and efficient energy management mechanism, the development of safe and efficient background management software.

\section{CONCLUDING REMARKS}

On the basis of deep research, the research and application of wireless sensor networks in agriculture are reviewed. First of all, to understand the wireless sensor network in detail, to understand the general architecture of wireless sensor network, which is composed of the sensing network, gateway, backbone network and user network. Secondly, introduces the application of wireless sensor networks in agriculture, and finally points out the existing wireless sensor network applications in agriculture based on the shortcomings, and puts forward specific solutions, pointed out the future direction of development. 


\section{REFERENCES}

[1] LIANG Zhujun, WU Li. Application of the environmental monitoring technology in the protected agriculture[J]. J Anhui Agric Sci, 2009, 37(16):7672 - 7673, 7753.

[2] ZHAO Jincai. Application analysis of measurement device in facility agriculture[J]. J Anhui Agric Sci, 2009, 37(16):5127 5128, 5206.

[3] XU Xichun, Chu Jiang, GAO Xiaohui. The development analysis of agricultural facilities [J]. J Agric Mech Res, 2008(8):237 - 240.

[4] BUTLER Z, CORKE P, PETERSON R, et al. Networked Cows: Virtual Fences for Con trolling Cows[EB/OL]. [2009-11-15].

[5] CAI Yihua, LIU Gang, LI Li, et al. Design and test of nodes for farmland data acquisition based on wireless sensor network[J]. Trans CSAE, 2009, 5(4):176-178 FLAMMINI A, FERRARI P, MARIOLI D, et al. Wired and wireless sensor networks for industrial applications Microelectron J, 2009, 40(9):1322 - 1336

[6] GAO Feng, YU Li, ZHANG Wenan, et al. Research and design of crop water status monitoring system based on wireless sensor networks[J]. Trans CSAE, 2009, 25(2) :107 - 112 